\title{
Engaging in Late-Life Mental Health Research: a Narrative Review of Challenges to Participation
}

Jordyn Newmark, $M S^{1}$

Marie Anne Gebara, $M D^{1, *}$.

Howard Aizenstein, MD, PhD ${ }^{1}$

Jordan F. Karp, $M D^{1,2}$

\author{
Address \\ ${ }^{*}, 1$ Department of Psychiatry, University of Pittsburgh School of Medicine, 3811 \\ 0'Hara St, Bellefield Towers 726, Pittsburgh, PA, 15213, USA \\ Email: gebarama@upmc.edu \\ ${ }^{2}$ Center for Interventions to Enhance Community Health, University of Pittsburgh, \\ Pittsburgh, USA
}

Published online: 22 May 2020

(C) Springer Nature Switzerland AG 2020

Keywords Barriers $\cdot$ Older adults $\cdot$ Mental health research

\begin{abstract}
Purpose of review This narrative review seeks to ascertain the challenges older patients face with participation in mental health clinical research studies and suggests creative strategies to minimize these obstacles.

Recent findings Challenges to older adults' engagement in mental health research include practical, institutional, and collaboration-related barriers applicable to all clinical trials as well as more personal, cultural, and age-related patient barriers specific to geriatric mental health research. Universal research challenges include (1) institutional barriers of lack of funding and researchers, inter-researcher conflict, and sampling bias; (2) collaborationrelated barriers involving miscommunication and clinician concerns; and (3) practical patient barriers such as scheduling issues, financial constraints, and transportation difficulties. Challenges unique to geriatric mental health research include (1) personal barriers such as no perceived need for treatment, prior negative experience, and mistrust of mental health research; (2) cultural barriers involving stigma and lack of bilingual or culturally matched staff; and (3) chronic medical issues and concerns about capacity. Summary Proposed solutions to these barriers include increased programmatic focus on and funding of geriatric psychiatry research grants, meeting with clinical staff to clarify study protocols and eligibility criteria, and offering transportation for participants. To minimize stigma and mistrust of psychiatric research, studies should devise community outreach efforts, employ culturally competent bilingual staff, and provide patient and family education about the study and general information about promoting mental health.
\end{abstract}




\section{Introduction}

Mental illness is increasingly prevalent worldwide, particularly in the aging population $[1,2]$. For those aged 65 years and older, 12-month prevalence rates are $11.4 \%$ for any anxiety disorder, $6.8 \%$ for any mood disorder, and 3.8\% for substance use disorder [3]. For older Medicare and Medicaid members, 12-month incidence rates are even higher at $19.4 \%$ for any behavioral health disorder and $11.2 \%$ for dementia [2]. However, approximately $70 \%$ of older adults with these disorders do not receive treatment [4].

Untreated depression and anxiety significantly decrease quality of life and increase risk of suicide and cognitive decline [5]. Older adults with major depressive disorder and comorbid generalized anxiety disorder or panic disorder also have greater disability and distress (including more severe somatic symptoms), poorer subjective health, and delayed acute response to treatment with poorer short-term treatment outcomes [5, 6]. Adults of all age groups with serious mental illness are four times as likely to have chronic obstructive pulmonary disease, twice as likely to have heart disease or diabetes, and almost five times as likely to have limitations in activities of daily living than those without serious mental illness [7].

Despite the prevalence of mental health disorders in older adults with associated comorbidities, increased severity of symptoms and disability, and higher health care utilization and costs, mental health research in older adults is not a national priority. In addition to programmatic and funding priorities which do not focus on older adults, clinical research in the geriatric population is confounded by age-related challenges. Identifying these modifiable challenges and developing creative and scalable solutions with broad uptake will assist late-life clinical researchers to conduct more clinically meaningful projects and lead to better implementation of evidence-based medicine.

Overcoming such barriers is essential to aid in recruitment and retention for mental health trials with older adults, which is a major problem: only $29 \%$ of clinical trials recruit within their original timeframe, while 56\% extend recruitment and one-third require additional funds $[8 \bullet]$. Consequences include increased costs and efforts, reduced statistical power of studies, and delays in generating evidence and subsequent adoption of effective interventions [9]. The purpose of this paper is to (1) describe identified barriers to older adult participation in clinical research and (2) recommend possible solutions to minimize these barriers that should be considered by clinical researchers as they develop projects. While the literature has been reviewed for barriers against recruitment for mental health studies pertaining to all ages [9-11], to our knowledge, there has been no review of barriers to recruitment and retention for geriatric mental health research. This narrative review will summarize findings from original studies and others to concisely present barriers for older adults and our proposed solutions to advance the field.

\section{Methods}

Search strategy

We searched the PubMed, EMBASE, and PsycINFO databases. Advanced searches were conducted using the terms "('mental health research' OR 'behavioral health research' OR 'psychiatric research') AND (participation OR recruitment OR retention) AND (older adults OR elder* OR aged OR aging OR geriatric)." Results were filtered by English language and age of subjects as $60+$, "adult," "middle aged," "aged," or "very elderly." Gray literature searches yielded additional relevant articles.

The search yielded 731 articles. After elimination of duplicate articles and a review of titles and abstracts, 26 papers were determined to fit the entry criteria "mental health research barriers specific to older adults." From PubMed, 6 of 431 articles were selected; from EMBASE, 9 of 280 articles; and from PsycINFO, 11 of 78 articles. Gray literature searches proved to be relevant and productive, yielding 7 additional papers for a total of 33 papers. All references were exported to Zotero. 
Although some barriers to research participation apply to all ages as well as older adults, this review focuses on geriatric patients, and thus, only papers on older adults or those comparing age groups were included. Likewise, many barriers to recruitment and retention for mental health clinical research apply to all clinical trials, and thus, articles specifying research topics unrelated to mental health were excluded.

\section{Results}

The thirty-three papers selected for inclusion in this review identified challenges in recruiting and retaining older adults for mental health clinical trials, and additional challenges faced in recruiting older minority populations for behavioral health research and treatment (Table 1). We subdivided these challenges into (1) practical, institutional, and researcher-clinician collaboration-related barriers pertaining to all clinical trials and (2) personal, cultural, and geriatricrelated patient barriers related to mental health research (Table 2).

\section{Institutional and study design barriers}

The need for aging-specific clinical research (evidenced by the growth of this age demographic and increasing life expectancy) exceeds the current number of researchers in geriatric psychiatry and is not aligned with the percent of the National Institute of Mental Health (NIMH) extramural budget allocated to geriatric mental health research $[16,35]$. Pressure to recruit more participants may result in negative attitudes and stressful environments detrimental to the mental health of research teams and their performance and recruitment efforts [43]. Misunderstandings and interdisciplinary conflict within research teams may be amplified in studies in which various professions differ in their academic languages and definitions of mental health conditions, leading to possible misdiagnoses or under-reporting of symptoms which may skew results [37].

Due to recruitment challenges, competition for participants across studies may be high, and research teams are often protective of their own "consent to be contacted" patient databases or primary care referral sources, limiting the pool of potential participants for other researchers [29, 43].Sampling bias further compounds this issue. Older adults who do not respond to recruitment efforts and survey studies or who tend to drop out of longitudinal studies differ from those who respond or continue. Recruitment non-responders/dropouts tend to have worse physical health and greater disabilities, report less physical activity, have fewer social contacts, lower education, poorer verbal skills, lower general intelligence, and be of lower socioeconomic class than respondents and those who complete studies [39]. As such, mental health studies unintentionally exclude more mentally ill patients who, due to the nature of their illness, are less likely to participate. This underrepresentation of a subset of mentally ill patients may lead to researchers overlooking or underestimating important correlates of mental illness. This phenomenon is commonly observed with depression, in which depression-associated ambivalence about joining a clinical trial, even if associated risks are minimal, limits participation. Depressed older adults are more likely than middle-aged and younger depressed adults to 


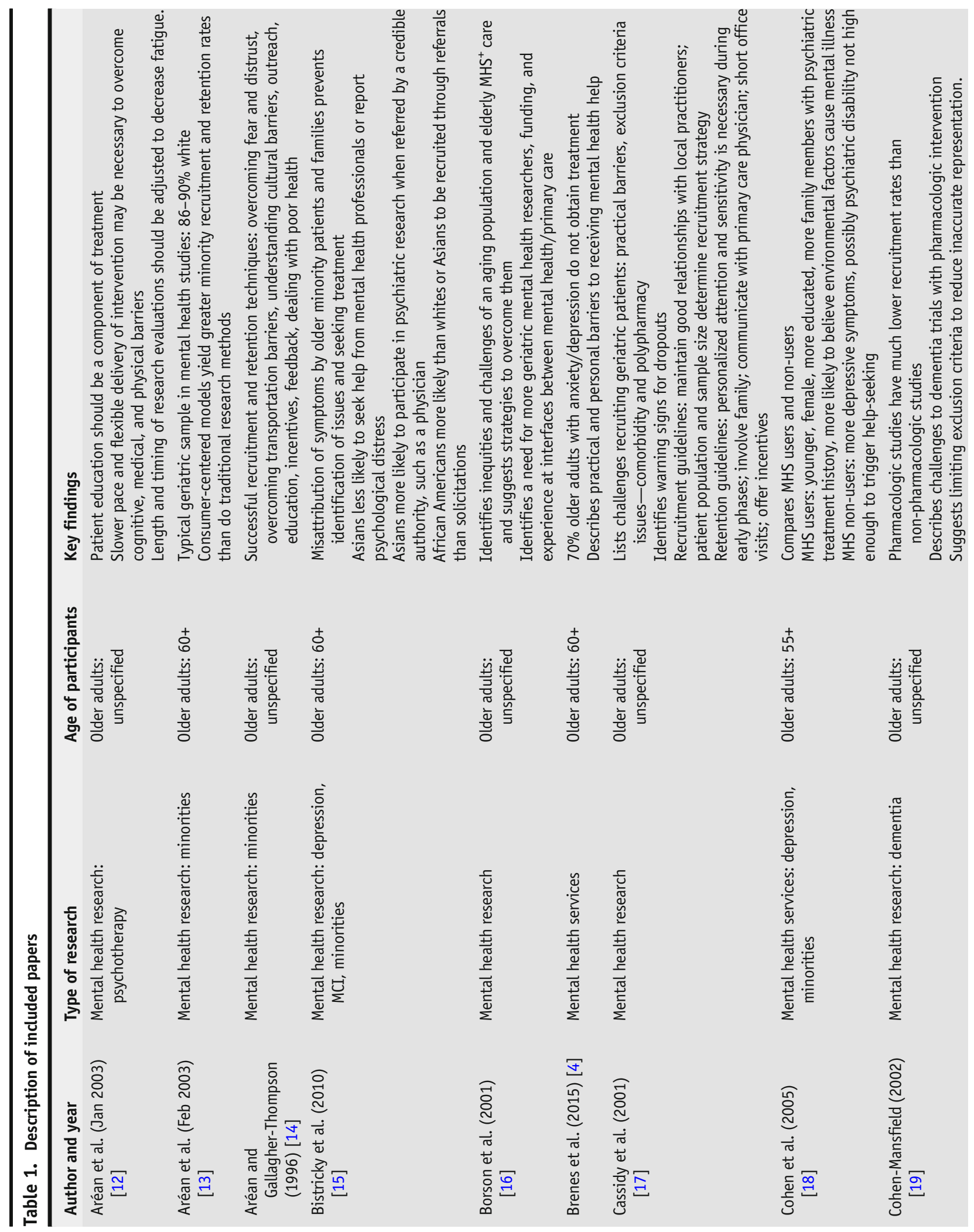




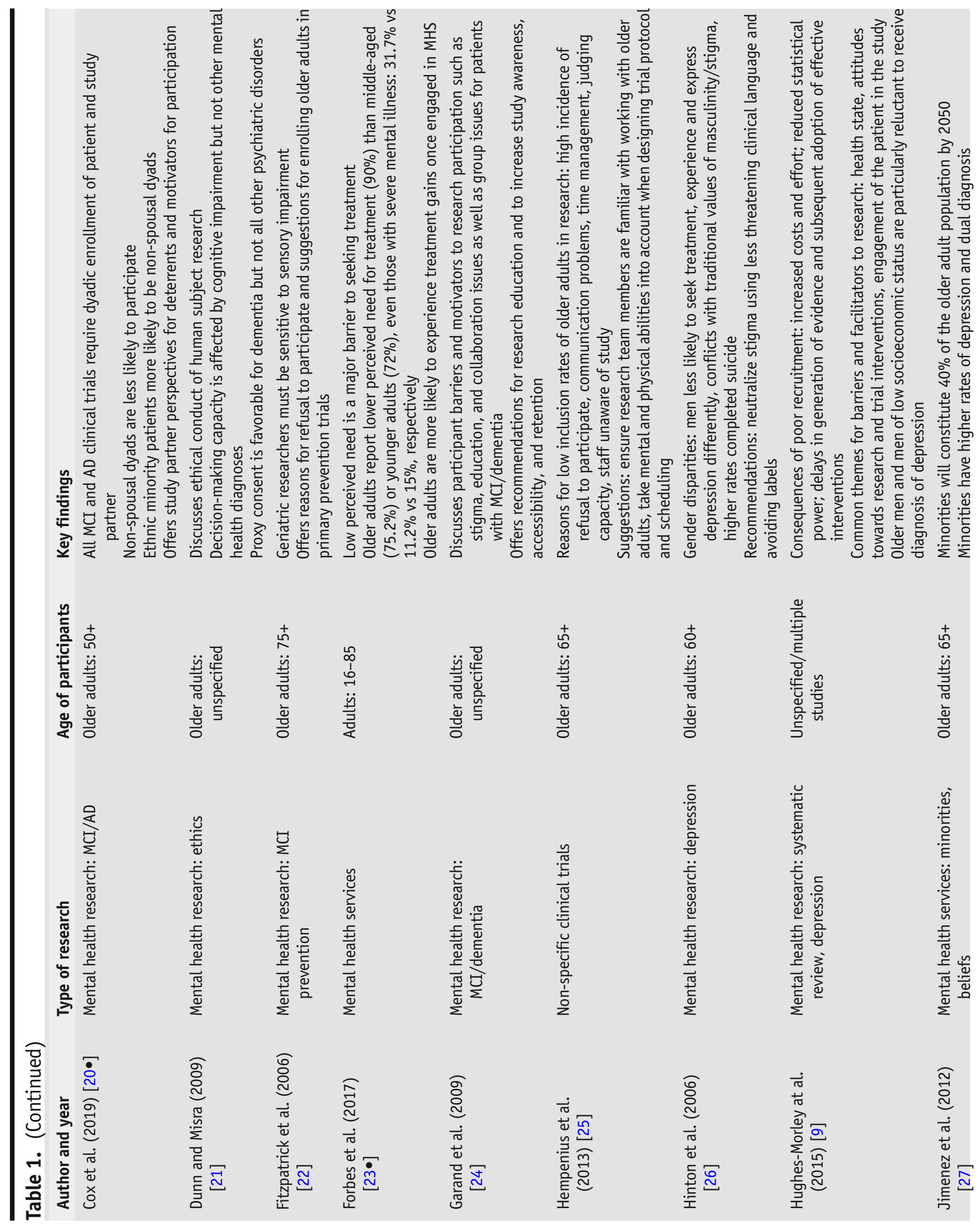




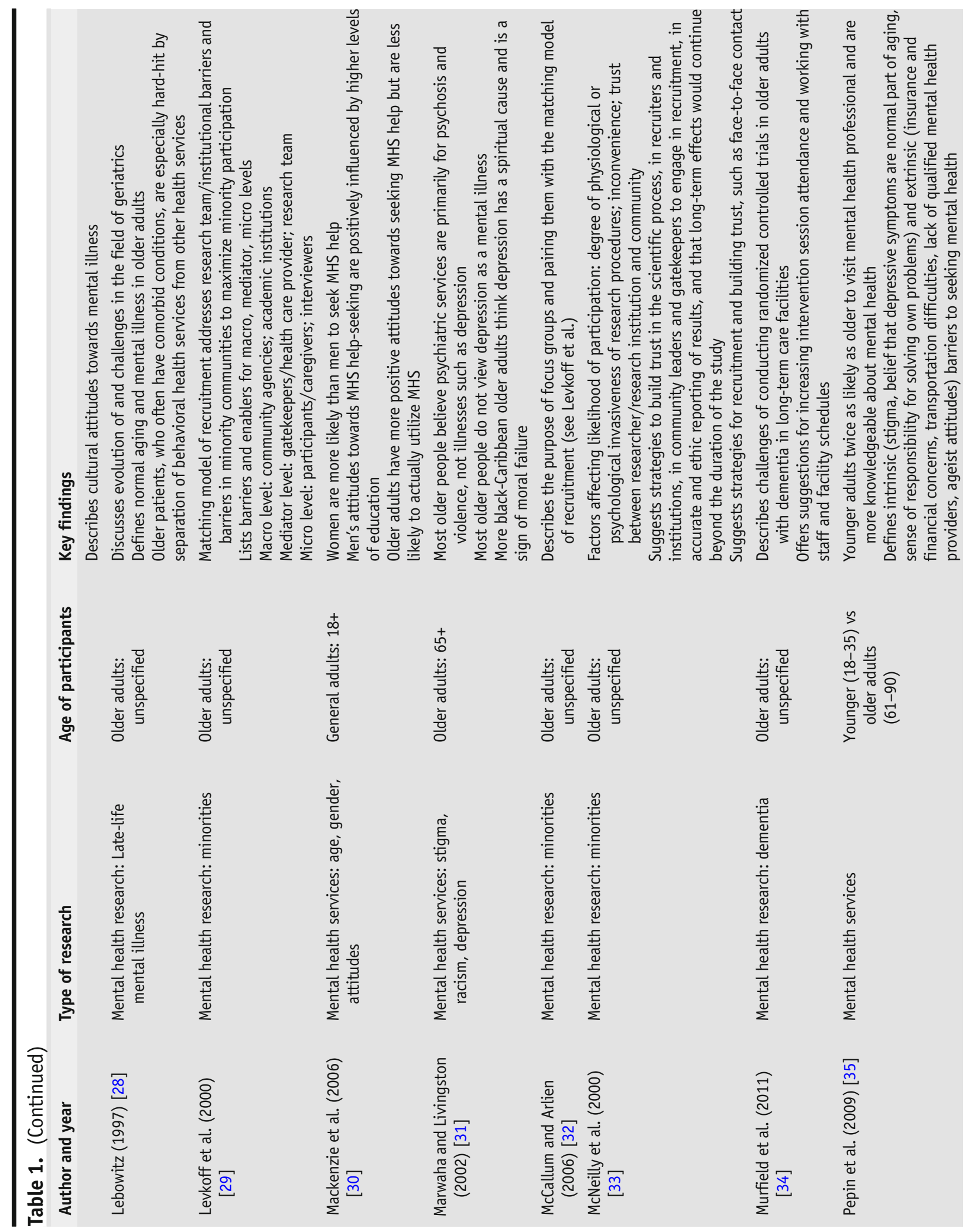




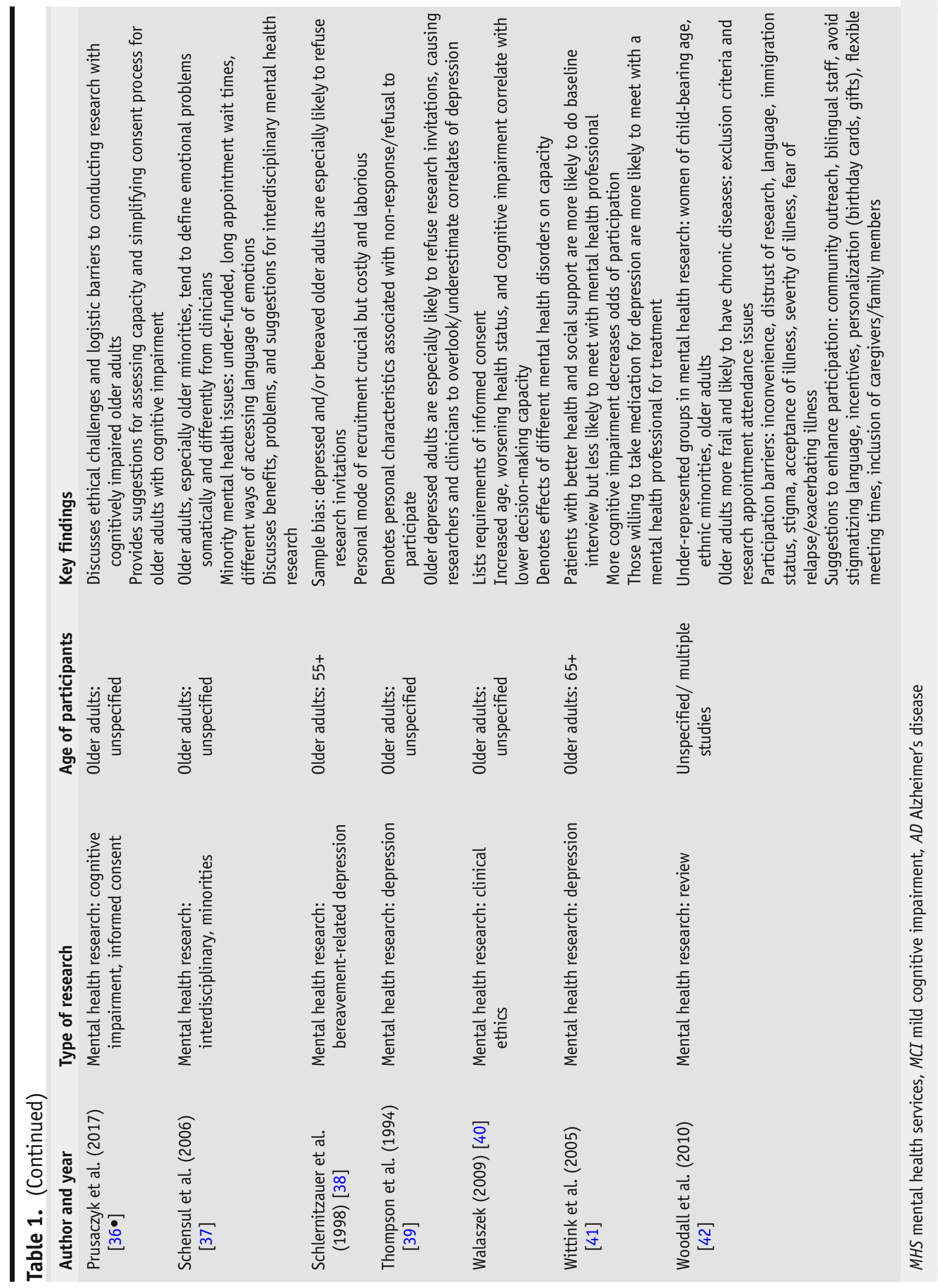


refuse to participate [39]. Patients with cognitive impairment, highly prevalent in depression, may further limit participation due to reduced decision-making capacity necessary to provide informed consent [40]. Thus, the nature of the mental illness itself may result in sampling bias and inaccurate representation of the disease being studied, an inherent challenge to clinical trials with this population.

\section{Collaboration-related barriers}

Clinical staff describe feeling overwhelmed with the burden of completing recruitment assessments and screening activities that do not account for their clinical responsibilities and workflow [44]. Clinical staff have reported feeling "used" by researchers for referrals, with their efforts going unrecognized and underappreciated $[45,46]$. These factors can engender unfavorable attitudes towards serving as community partners and sources of patient participants for clinical research studies [47].

Clinical physician community partners can interfere with referrals and participation of their patients. They describe concerns about loss of autonomy over the patient's care, how research will affect the doctor-patient relationship, and potential side effects, and the burden of research, especially for very old or severely ill patients $[48,49]$. While these decisions are motivated by caring, this overprotectiveness of patients leads some physicians to assume decisionmaking on behalf of some patients who may have been eligible and interested in participating $[45,50,51]$. Community physician partners may hesitate to introduce a trial if they are unclear about eligibility criteria, trial protocol, or the purpose of the study, or if they feel that there is a lack of equipoise in the study arms or a risk that their ill patients may be exposed to placebo [50].

\section{Practical barriers}

Practical barriers are ubiquitous to all potential participants: this includes time commitment, interference with work schedules, lack of health insurance that may prevent access to mental health services for referrals, and transportation difficulties [4]. The latter of these is especially troublesome for older adults who may not be able to drive, for those of lower socioeconomic class who often rely on public transportation, and for minorities concerned of becoming victims of racism if the research center is in an area outside their community [14, 35]. At the time of preparing this manuscript, efforts to mitigate the Coronavirus pandemic of 2019-2020 include self-isolation and avoiding health care centers which contain a higher density of symptomatic patients and are often the same locations where clinical research is conducted [52]. Although not further discussed in this manuscript, these mitigation efforts are currently an obstacle to ongoing clinical trials, and funders and researchers are rapidly developing and adopting clinical procedures that may be conducted remotely to minimize the impact on clinical research of these contagion mitigation strategies.

Older adults are significantly more likely than middle-aged or younger adults to perceive no need for mental health treatment, even among those with serious mental illness [23•]. Furthermore, some patients may have unfavorable attitudes towards mental health research from prior negative experiences with 


\section{Table 2. Barriers to older adults participating in mental health clinical trials}

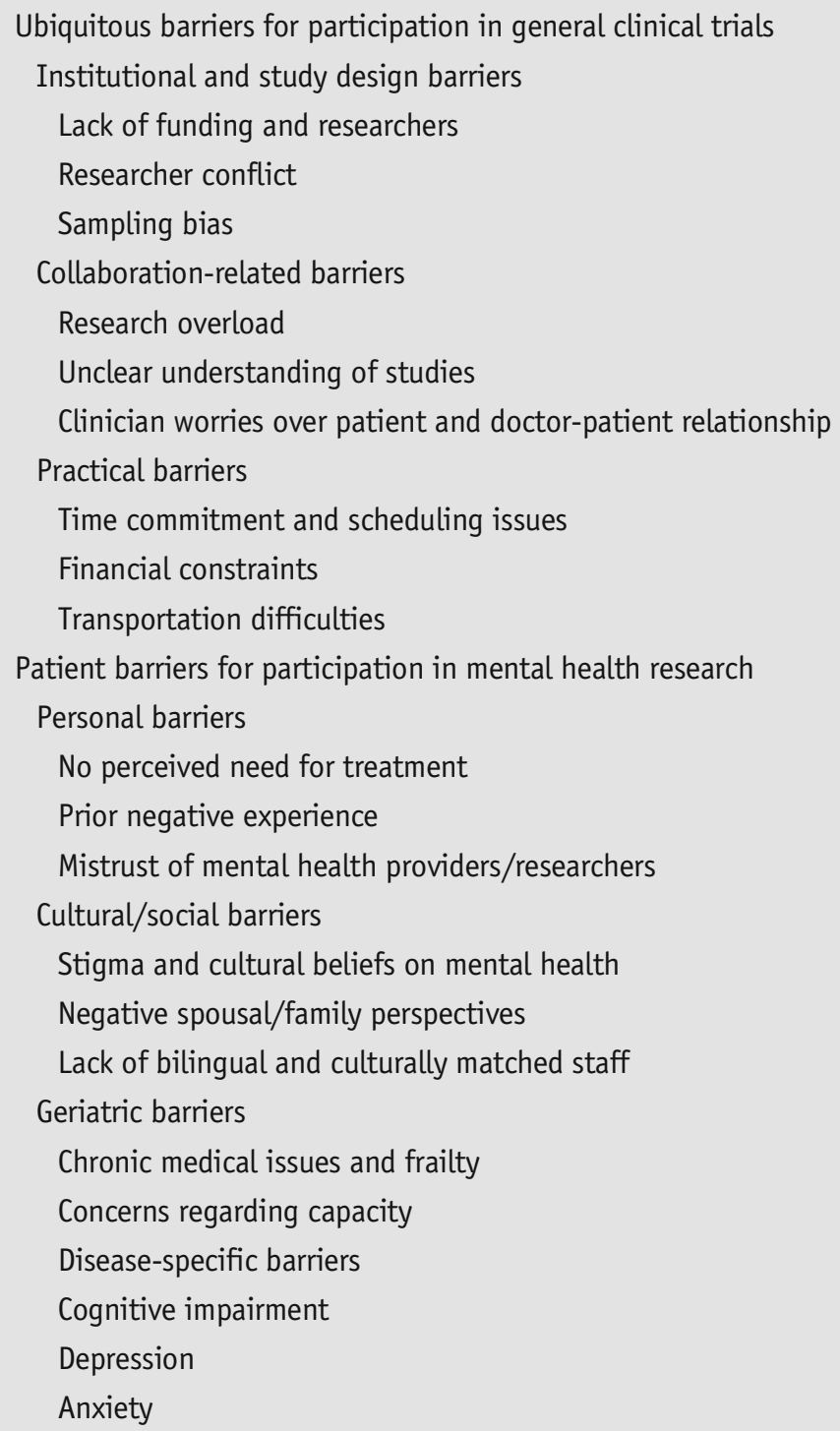

mental health treatment, especially if the study proposes a similar intervention (i.e., medication or psychotherapy) to one they have already tried [9]. Other personal barriers include mistrust of mental health providers or researchers. While mistrust or fear of medical research is a barrier to research afflicting all populations, it is especially pronounced in minorities due to historical unethical research practices such as the Tuskegee syphilis study [4, 10, 20•, 53]. Racial and ethnic minorities are at higher risk of mental illness, yet they tend to underutilize behavioral health services and are chronically under-represented in mental health research [54]. 


\section{Table 3. Recommendations on increasing participation in geriatric mental health research}

Addressing ubiquitous barriers for participation in general clinical trials

Institutions and funders/sponsors

Improve support of clinician-researchers through protected research time

Incorporate PBRNs to link research with clinical practice

Research team with clinical collaborators

Use common terms to facilitate communication in multidisciplinary studies

Acknowledge and express appreciation for all members of research team

Establish a research presence at clinical sites

Offer research training for the clinical team

Maintain frequent contact with referring clinicians and provide feedback and study updates

Address clinician concerns regarding placebo

Use comparison arms with active treatments

Educate clinicians on likelihood for improvement in study regardless of randomization arm

Researchers involved in study design

Offer flexible scheduling including evening and weekend appointments

Implement electronic consent procedures

Provide financial compensation

Offer transportation assistance

Conduct research in the community instead of/in addition to university settings

Addressing barriers for participation in geriatric mental health research

Institutions and funders/sponsors

Increase supplements and training grants for geriatric psychiatry research

Institute collaborative hubs for expanding mental health research in low- to middle-income countries

Support more interventional studies to increase participation in mental health research

Sponsor community events and lectures to raise awareness for mental health and mental health research

Research team with community

Employ bilingual or culturally matched research team members involved in recruitment

Offer cultural literacy training for research staff

Offer mental health training to primary care providers

Reach out to community leaders, faith leaders, senior centers for mental health awareness and education

Involve community members in study design and recruitment

Implement research advocacy training programs

Researchers with potential participants

Attenuate exclusion criteria regarding comorbidities and polypharmacy for more "real world" sample and greater eligibility Limit consultation time and shorten assessment visits

Accommodate physical impairments by having large-print documents and voice amplifiers available

Use PowerPoint presentations and summaries of relevant information to enhance comprehension

Clarify study protocols and voluntary nature of study to increase participant sense of autonomy

Emphasize study purpose and potential benefits to appeal to altruistic tendencies 
Table 3. (Continued)

Have caregiver/family member present during consent process Offer patient and family psychoeducation

PBRNs practice-based research networks

Perceived stigma is a universal barrier to engaging in mental health treatment and research, especially among older adults. Fear of public stigma or the individual's own stereotypes about mental health (self-stigma) may reduce willingness of potential participants to learn about available research studies [24]. Older whites are often concerned about how a mental health diagnosis will affect others' perceptions of them; older minorities may worry more about how such a diagnosis will impact their family's reputation [13].

Mental illness stigma is more pronounced among older adults, who tend to have higher thresholds for what constitutes mental illness as opposed to "normal aging" and what symptoms merit treatment [23•]. They may avoid seeking treatment or agreeing to participate in studies on mental health for fear that a mental health diagnosis indicates they are "crazy." [24] These negative views may be most prominent in the very-old who associated mental illness with psychosis, viewed with fear or seen as a personal flaw [31].This is especially relevant among older men for whom mental illness may be viewed as a weakness and emotional vulnerability that conflicts with traditionally "masculine" values of strength, stoicism, and internalizing one's emotional problems [26]. Older men may be more likely to express depression through somatic symptoms, anger, and substance use, rather than describing emotional distress, making case identification for treatment or research more challenging [26].

Cultural stigma may discourage older minorities from seeking mental health treatment or enrolling in psychiatric research studies. For example, Asians are less willing to participate in psychiatric clinical research compared with other racial and ethnic groups, partially attributed to cultural views of health and health care that conflict with those of Western medicine and to the belief that a mental health diagnosis would stigmatize their family $[15 \bullet, 29,55]$.Older ethnic minorities in Western cultures are more likely than older Caucasians to have stereotyped negative ideas about mental illness that interferes with research participation [14].

Cultural and language barriers further complicate participation by contributing to communication difficulties between the potential participant and research team $[10,29,54]$.Cultural traditions may affect how recruitment and research assessments are performed. For instance, Confucian ethical rules conferring decision-making to the eldest male family member may necessitate recruiters of an older female Chinese participant to contact her uncle or older brother for consent to participate [29]. For immigrants and older minorities, limited literacy, lack of culturally adapted questionnaires, and alarm-inducing phrases from translation gaps may contribute to many older adults refusing to participate in research [10, 41]. Immigrants may be especially suspicious of the consent process and signing legal documents, as they may have concerns that participating in research will affect legal residency status and possible deportation $[10,29,56]$. 
Older adults may be more physically frail and more likely to have chronic physical diseases which can limit clinical trial participation because of exclusion criteria and participant burden [42]. Polypharmacy related to comorbid medical conditions is a common reason older patients are excluded from participation in trials [17]. Some frail older adults are concerned that perceived physical or psychosocial stressors from study participation would make them fatigued or worsen other medical conditions. For instance, older adults who have learned to cope with their depression may fear that changes in their treatment or daily routine resulting from trial participation may disrupt their ability to maintain routines and manage their medical and mental illnesses [9].

Some frail older adults do not enroll in clinical trials due to communication problems, physical and cognitive limitations, and feeling overburdened by their physical conditions and/or do not want to be a burden to relatives [25]. Hempenius et al. (2013) observed that older adults' communicative capacity is often restricted by sensory loss, speech problems, and cognitive decline. These communication difficulties can slow the informed consent process and adherence to study protocols [25]. Mild cognitive impairment, Alzheimer's disease, and other dementias may impair ability to provide truly informed consent [21, 40]. Indeed, greater cognitive impairment is associated with decreased odds of study participation [41].

Older adults with cognitive impairment are often purposefully excluded from research not related to cognition due to concerns about informed consent [36•]. For older adults with severe cognitive impairment, proxy consent involving next of kin is almost always required. This can pose issues for recruitment, as family members may believe that their relative's health status would preclude participation [34]. Conversely, families may desire their relative to participate only in the intervention and not the control activity of the study, which would disrupt randomization and study integrity [34].

Engaging older adults in psychiatric clinical trials is often made more difficult due to the nature of the mental illness itself. In addition to complications related to capacity and consent for older adults with cognitive impairment or dementia, the level of cognitive decline and presence of agitated behaviors may affect or confound implementation of study interventions [34]. Seniors with depression, who are more likely to suffer from decreased energy and lack of motivation, report more perceived barriers to psychological treatment, greater stigma, and are more likely to negatively evaluate therapy interventions than non-depressed adults [57]. Older adults with anxiety often interpret information in a negative or threatening manner [4]. Therefore, some may be more fearful of study interventions and potential side effects, contributing to participation refusal and withdrawal.

Just as some barriers to participation in geriatric mental health research are relevant to all clinical trials while others are specific to the field of geriatric psychiatric research, so too are the potential solutions to address these concerns, summarized in Table 3. 
Some of the barriers contributing to this lack of participation in geriatric psychiatric research are ubiquitous to all clinical trials, including institutional and study design barriers, collaborative barriers, and practical barriers to accessing studies. Institutional barriers include the relative lack of funding and researchers to support studies in geriatric psychiatry. Increasing supplements and training grants to support postdoctoral fellowships in geriatric mental health research may enhance the pool of geriatric psychiatry researchers and studies [16]. This is especially important in low- to middle-income countries (LMICs) in which the lack of trained mental health researchers limits progress in global mental health research [58]. Capacity-building initiatives, such as the US National Institute of Mental Health Collaborative Hubs for International Research on Mental Health, have been implemented to provide the knowledge and tools to increase research capacity in LMICs [58]. Collaborations through research hubs in more industrialized countries can help with implementation of evidence-based practices for mental health in these LIMCs while simultaneously expanding the knowledge base of global mental health.

More generally, encouraging institutional changes supporting the role of clinician-researchers, such as protected research time, may reduce clinical workloads and facilitate more research activity [43]. Additionally, facilitating the development of practice-based research networks (PBRNs) and coordination with academic centers may enhance community recruitment and implementation and dissemination of study findings into clinical practice [59, 60].

Improving interpersonal harmony within and across multidisciplinary research teams may improve productivity and success of clinical trials. To minimize conflicts within multidisciplinary studies involving academic researchers and community staff such as social workers, primary care physicians, and nurses, clinical terms and principles specific to each discipline should be defined and translated into common terms. Additionally, acknowledging the efforts of the entire research team, from individuals recruiting in the community to those analyzing the data, creates unity and a more positive environment to facilitate clinical and research workplace efficiencies $[45,46]$. Gratitude to other team members can be conveyed through verbal expressions of appreciation, cards or electronic correspondence, acknowledgement in the publication, or, when appropriate, offering publication opportunities.

To enhance relationships between researchers and clinical staff, it may be helpful to establish a research presence onsite in clinical settings, maintain frequent contact with referring clinicians, and provide feedback and study updates with preliminary data to retain interest in the study. These efforts have been shown to improve referral rates [14, 43, 45]. Issues arising from lack of communication between the clinical and research teams highlight the importance of reviewing relevant study information, offering training for the clinical team, and providing opportunities to answer questions [46]. These efforts enable referring clinicians to feel more confident introducing studies to patients and reduce misunderstandings and miscommunications about study aims and protocols.

To alleviate physicians' concerns about their older patients engaging in research or being exposed to placebo, researchers should consider using comparison arms with active treatments as opposed to placebo. Alternately, 
researchers should emphasize the increased likelihood for improvement regardless of randomization arm. Simply participating in a study may benefit older patients with psychiatric illness, as additional follow-up visits have been shown to improve outcomes for both placebo (by 41\%) and antidepressants (by 27\%) [61]. Indeed, participants in randomized clinical trials receiving a placebo are more likely to experience improvement in their depressive symptoms than patients receiving antidepressant treatment under a primary care physician's usual care [62]. This may be due in part to differences in the process of care provided in a protocolized clinical trial; patients receiving usual care from a community-based provider are less likely to receive measurement-based care and as frequent follow-ups as those participating in a clinical trial [62, 63].

The more universal patient-related barriers to engaging in research are of a practical nature: this includes financial, scheduling, time commitment, and transportation concerns. These can be alleviated by offering flexible scheduling including evening and weekend appointments, implementing electronic consent procedures, using video and telephonic technology to assess and monitor research participants, providing financial compensation for study participation, and offering parking vouchers, taxi/shuttle services, reimbursement for transportation costs, and conducting research procedures beyond the university and instead in community settings $[14,25,42,54]$.

\section{Disease-related, geriatric, personal, and social barriers}

Concerns about engaging older adults in research include chronic medical conditions, frailty, and ability to consent. In consideration of decreased physical and cognitive reserve, adaptations include limiting consultation time and shortening assessment visits, communicating with older patients face-to-face rather than by phone to enhance information comprehension and retention, having large-print documents available for those with visual impairments, and accommodating those with other physical impairments such as voice amplifiers for patients with hearing impairments [25]. For patients with multiple medical conditions, communicating with referring primary care providers to verify medical information and confirming there are no contraindications for study eligibility will promote the safety of participants and engages referring physicians in the research process [25]. Given the commonality of medical comorbidities in older adults, researchers should consider attenuating exclusion criteria relating to comorbidities and polypharmacy to improve study eligibility and encompass a study population that is more representative of "real world" older adults.

Having a caregiver present while initiating the informed consent conversation can ease anxiety about the consent procedure and remind the participant of any forgotten information [25]. Additionally, it is helpful to use PowerPoint presentations and summaries of relevant information that have been shown to enhance comprehension of consent for research in older patients with psychosis [64]. As individuals with mental health diagnoses are differentially affected by their disease and some may maintain more insight and greater capacity to consent than others with the same disease, it may be necessary to formally assess capacity prior to obtaining informed consent $[21,36 \bullet]$. 
Other barriers to older patients engaging in mental health research are related to mistrust of research, misunderstanding of mental health conditions, stigma, and cultural and language barriers. In efforts to alleviate mistrust in researchers and the research process, it is important to clearly communicate study aims, protocols, and the consent process. Older adults are often motivated by altruism and a desire to improve the world for future generations $[8 \bullet, 9,20 \bullet, 24,45,46,65,66]$, so care should be taken to emphasize the purpose of the study and how it can advance knowledge of and treatment for the mental health issue being investigated to help others in the future. Since patients highly value their sense of autonomy, researchers should stress the voluntary nature of participation in the study and ability to withdraw at any time that is consistent with the Belmont Report [66]. Keeping the patient and family apprised of the study progress and sharing study findings further solidifies trust and may promote retention [67].

Researchers should emphasize the symptoms being targeted in trials, teach participants about minimizing stress, and refrain from using stigmatizing diagnostic labels $[24,26]$. To prevent miscommunication in patients with low literacy, it is preferable to describe procedures rather than to use technical jargon [42] and attempt to keep written materials at sixth grade level of literacy $[68,69]$. Employing multilingual, multi-cultural staff for conducting interviews, participating in the informed consent process, and reviewing documentation to avoid stigma- or alarm-inducing phrases and translational errors is crucial for working with older minorities or multilingual populations to enable them to feel comfortable and able to communicate effectively [10,42]. Researcher selection and cultural competency training can compensate for lack of ethnically matched staff to comprise a research team that is sensitive to participants' needs [54].

\section{Patient, family, and community education}

Education on mental health conditions and treatments is associated with more positive perspectives on mental health, whereas misconceptions regarding mental illness lead to failure to recognize symptoms and perseverance of negative attitudes [12•]. Offering thorough education on mental health conditions and on the requirements and benefits of the proposed research study may minimize confusion and encourage higher participation rates. Using personal stories to illustrate potential research benefits facilitates patient rapport and can provide an experience enabling participants to feel more open and trusting [65].

Extending patient education to the family and caregivers to actively involve them in recruitment and research procedures has been shown to increase study participation rates, as the decision to consent is largely influenced by close relations $[20 \bullet, 42]$.Family/caregiver education is particularly important for patients with dementia or questionable decision-making capacity secondary to their condition. Additionally, as caregivers of older adults often experience elevated stress, anxiety, and depression, researchers should inquire about the mental health status of caregivers and provide appropriate resources [28]. Addressing caregiver mental health concerns enables them to better perform their responsibilities as a caregiver and may improve their overall attitude towards mental health and the research process. An interventional study offering psychoeducational workshops to families of older patients with depression 
supports this theory, noting a lower rate of dropout during continuation of treatment and suggesting that "psychoeducation and support may be especially important for families of elderly patients with depression who may be faced with an increase in an already substantial caretaking burden." [70]

Community outreach efforts to establish relationships with geriatric and older adult minority communities can help promote positive perceptions about mental health treatment and research. Sponsoring community events and lectures, serving as guest expert on radio programs, and providing written and durable electronic information materials about mental health and research at senior centers, churches, and other community centers can establish the presence of researchers in the community, remove the mystery surrounding research to make it seem more like an opportunity, and provide a forum for older adults to ask questions about research methods and ethics [33]. Research advocacy training programs for older adults increase the likelihood of participation in research and advocating for future studies [71]. As the majority of elderly persons receiving mental health care are treated by their general medical providers, it is essential to teach basic geriatric mental health skills to current and future primary care providers as well [16]. Dowrick et al. (2016) implemented an interventional study linking primary care training with community engagement in an Improving Access to Mental Health in Primary Care (AMP) model that also incorporated a wellbeing intervention based on cognitive-behavioral principles. They found that community engagement (including housing associations, faith leaders, police, business leaders, and health practitioners) raised awareness of mental health issues and increased referrals to the wellbeing intervention, while recruitment was positively associated with offering training to primary care providers [72]. Kogan et al. (2009) also emphasize the importance of including community partners from the outset in the process of research development and implementation with involving minority groups in bipolar disorder research, noting $45 \%$ enrollment from minority populations at community sites compared with $15 \%$ at academic sites [73]. The few studies exploring specific interventions to increase participation in mental health research illustrate success with implementing community outreach and primary care training efforts, but these studies are limited and demonstrate the need for additional interventional research to guide how researchers approach recruitment for mental health studies in the future.

\section{Conclusions}

Since the burden of mental illness prevalence in older adults is disproportionate to research opportunities and engagement, efforts to reduce barriers to research participation are critical. Primary limiting factors for participation in geriatric mental health research are practical and personal barriers including inaccessibility, stigma, lack of education regarding mental health issues, perceived lack of benefit from research, and mistrust of research studies. Promising approaches to address these limitations include enhancing engagement across the research team and the community. Specific strategies include increasing PCP knowledge about available studies and improved integration with psychiatric services; 
community outreach through community events and both traditional and social media; having culturally competent and bilingual members of research staff; and providing both patient and family/caregiver education about mental health conditions and the purpose and procedures of the studies to assure families support the participation of their older relatives.

\section{Compliance with Ethical Standards}

\section{Conflict of Interest}

Dr. Karp reports receipt of medication supplies for investigator-initiated studies from Pfizer and Indivior. He receives compensation for editorial work for the American Journal of Geriatric Psychiatry and the Journal of Clinical Psychiatry and has received an honorarium from Otsuka for a disease-state medical education webinar. The other authors report no conflicts with any product mentioned or concept discussed in this article. Ms. Newmark is supported by NIMH T32 MH 19986.

\section{References and Recommended Reading}

Papers of particular interest, published recently, have been highlighted as:

- Of importance

1. Andresen EL, Wilson KA, Castillo A, Koopman C. Patient motivation for participating in clinical trials for depression: validation of the motivation for clinical trials inventory-depression. Int Clin Psychopharmacol. 2010;25(1):7-16. https://doi.org/10.1097/YIC. 0b013e328332055c.

2. Lin W-C, Zhang J, Leung GY, Clark RE. Twelve-month diagnosed prevalence of behavioral health disorders among elderly Medicare and Medicaid members. Am J Geriatr Psychiatry. 2011;19(11):970-9. https://doi. org/10.1097/JGP.0b013e3182011b66.

3. Reynolds K, Pietrzak RH, El-Gabalawy R, Mackenzie CS, Sareen J. Prevalence of psychiatric disorders in U.S older adults: findings from a nationally representative survey. World Psychiatry. 2015;14(1):74-81. https:// doi.org/10.1002/wps.20193.

4. Brenes GA, Danhauer SC, Lyles MF, Hogan PE, Miller ME. Barriers to mental health treatment in rural older adults. Am J Geriatr Psychiatry. 2015;23(11):1172-8. https://doi.org/10.1016/j.jagp.2015.06.002.

5. DeLuca AK, Lenze EJ, Mulsant BH, et al. Comorbid anxiety disorder in late life depression: association with memory decline over four years. Int J Geriatr Psychiatry. 2005;20(9):848-54. https://doi.org/10.1002/gps. 1366.

6. Cairney J, Corna LM, Veldhuizen S, Herrmann N, Streiner DL. Comorbid depression and anxiety in later life: patterns of association, subjective well-being, and impairment. Am J Geriatr Psychiatry. 2008;16(3):2018. https://doi.org/10.1097/01.JGP.0000300627. 93523.c8.
7. Weissman J. Serious psychological distress among adults: United States, 2009-2013. 2015;(203):8.

8. Tallon D, Mulligan J, Wiles N, et al. Involving patients with depression in research: survey of patients' attitudes to participation. $\mathrm{Br} J$ Gen Pract. 2011;61(585):e134-41. https://doi.org/10.3399/ bjgp $11 \times 567036$.

Relevant systematic reviews on recruitment and retention in mental health research.

9. Hughes-Morley A, Young B, Waheed W, Small N, Bower P. Factors affecting recruitment into depression trials: systematic review, meta-synthesis and conceptual framework. J Affect Disord. 2015;172:274-90. https://doi.org/10.1016/j.jad.2014.10.005.

10. Brown G, Marshall M, Bower P, Woodham A, Waheed W. Barriers to recruiting ethnic minorities to mental health research: a systematic review. Int J Methods Psychiatr Res. 2014;23(1):36-48. https://doi.org/10. 1002/mpr.1434.

11. Liu Y, Pencheon E, Hunter RM, Moncrieff J, Freemantle N. Recruitment and retention strategies in mental health trials - a systematic review. PLoS ONE. 2018;13(8). doi:https://doi.org/10.1371/journal. pone.0203127

12.• Areán PA, Cook BL, Gallagher-Thompson D, Hegel MT, Schulberg HC, Schulz R. Guidelines for conducting geropsychotherapy research. Am J Geriatr Psychiatry. 2003;11(1):9-16. https://doi.org/10.1097/ 00019442-200301000-00003.

Issues and recommendations for engaging older minorities in mental health clinical research. 
13. Areán PA, Alvidrez J, Nery R, Estes C, Linkins K. Recruitment and retention of older minorities in mental health services research. The Gerontologist. 2003;43(1):36-44. https://doi.org/10.1093/geront/ 43.1.36

14. Areán PA, Gallagher-Thompson D. Issues and recommendations for the recruitment and retention of older ethnic minority adults into clinical research. J Consult Clin Psychol. 1996;64(5):875-80. https://doi.org/10. 1037/0022-006X.64.5.875.

15. Bistricky SL, Mackin RS, Chu JP, Areán PA. Recruitment of African Americans and Asian Americans with latelife depression and mild cognitive impairment. Am J Geriatr Psychiatry. 2010;18(8):734-42. https://doi.org/ 10.1097/JGP.0b013e3181cc0314.

Cognitive impairment, informed consent, and geriatric issues in the conduction of mental health clinical trials in older adults.

16. Borson S, Bartels SJ, Colenda CC, Gottlieb GL, Meyers B. Geriatric mental health services research: strategic plan for an aging population: report of the Health Services Work Group of the American Association for Geriatric Psychiatry. Am J Geriatr Psychiatry. 2001;9(3):191-204. https://doi.org/10.1097/ 00019442-200108000-00002.

17. Cassidy EL, Baird E, Sheikh JI. Recruitment and retention of elderly patients in clinical trials: issues and strategies. Am J Geriatr Psychiatry. 2001;9(2):136-40. https://doi.org/10.1097/00019442-20010500000005.

18. Cohen CI, Magai C, Yaffee R, Walcott-Brown L. Comparison of users and non-users of mental health services among depressed, older, urban African Americans. Am J Geriatr Psychiatry. 2005;13(7):545-53. https://doi.org/10.1097/00019442-20050700000002.

19. Cohen-Mansfield J. Recruitment rates in gerontological research: the situation for drug trials in dementia may be worse than previously reported. Alzheimer Dis Assoc Disord. 2002;16(4):279-82.

20. Cox CG, Ryan BA, Mary M, Gillen DL, Grill JD. A preliminary study of clinical trial enrollment decisions among people with mild cognitive impairment and their study partners. Am J Geriatr Psychiatry. 2019;27(3):322-32. https://doi.org/10.1016/j.jagp. 2018.10.016.

Cognitive impairment, informed consent, and geriatric issues in the conduction of mental health clinical trials in older adults.

21. Dunn LB, Misra S. Research ethics issues in geriatric psychiatry. Psychiatr Clin North Am. 2009;32(2):395411. https://doi.org/10.1016/j.psc.2009.03.007.

22. Fitzpatrick AL, Fried LP, Williamson J, Crowley P, Posey D, Kwong L, et al. Recruitment of the elderly into a pharmacologic prevention trial: the Ginkgo Evaluation of Memory Study experience. Contemp Clin Trials. 2006;27(6):541-53. https://doi.org/10.1016/j.cct. 2006.06.007.
23. Forbes MK, Crome E, Sunderland M, Wuthrich VM. Perceived needs for mental health care and barriers to treatment across age groups. Aging Ment Health. 2017;21(10):1072-8. https://doi.org/10.1080/ 13607863.2016.1193121.

Cognitive impairment, informed consent, and geriatric issues in the conduction of mental health clinical trials in older adults.

24. Garand L, Lingler JH, Conner KO, Dew MA. Diagnostic labels, stigma, and participation in research related to dementia and mild cognitive impairment. Res Gerontol Nurs. 2009;2(2):112-21. https://doi.org/10. 3928/19404921-20090401-04.

25. Hempenius L, Slaets JPJ, Boelens MAM, van Asselt DZB, de Bock GH, Wiggers T, et al. Inclusion of frail elderly patients in clinical trials: solutions to the problems. J Geriatr Oncol. 2013;4(1):26-31.

26. Hinton L, Zweifach M, Tang L, Unützer J, Oishi S. Gender disparities in the treatment of late-life depression: qualitative and quantitative findings from the IMPACT trial. Am J Geriatr Psychiatry. 2006;14(10):884-92. https://doi.org/10.1097/01.JGP. 0000219282.32915.a4.

27. Jimenez DE, Bartels SJ, Cardenas V, Dhaliwal SS, Alegría M. Cultural beliefs and mental health treatment preferences of ethnically diverse older adult consumers in primary care. Am J Geriatr Psychiatry. 2012;20(6):533-42. https://doi.org/10.1097/JGP. ob013e318227f876.

28. Lebowitz BD. The future of clinical research in mental disorders of late life. Schizophr Res. 1997;27(2):2617. https://doi.org/10.1016/S0920-9964(97)00064-9.

29. Levkoff SE, Levy BR, Weitzman PF. The matching model of recruitment. J Ment Health Aging. 2000;6(1):29-38.

30. Mackenzie CSP, Gekoski WL, Knox VJ. Age, gender, and the underutilization of mental health services: the influence of help-seeking attitudes. Aging Ment Health. 2006;10(6):574-82. https://doi.org/10.1080/ 13607860600641200.

31. Marwaha S, Livingston G. Stigma, racism or choice. Why do depressed ethnic elders avoid psychiatrists? J Affect Disord. 2002;72(3):257-65. https://doi.org/10. 1016/S0165-0327(01)00470-0.

32. McCallum TJ, Arlien CR. Enhancing the matching model of recruitment through focus groups. Aging Ment Health. 2006;10(3):312-8. https://doi.org/10. 1080/13607860500409781.

33. McNeilly M, Musick M, Efland JR, et al. Minority populations and psychophysiologic research: challenges in trust building and recruitment. J Ment Health Aging. 2000;6(1):91-102.

34. Murfield J, Cooke M, Moyle W, Shum D, Harrison S. Conducting randomized controlled trials with older people with dementia in long-term care: challenges and lessons learnt. Int J Nurs Pract. 2011;17(1):52-9. https://doi.org/10.1111/j.1440-172X.2010.01906.x.

35. Pepin R, Segal DL, Coolidge FL. Intrinsic and extrinsic barriers to mental health care among community- 
dwelling younger and older adults. Aging Ment Health. 2009;13(5):769-77. https://doi.org/10.1080/ 13607860902918231.

36. Prusaczyk B, Cherney SM, Carpenter CR, DuBois JM. Informed consent to research with cognitively impaired adults: transdisciplinary challenges and opportunities. Clin Gerontol. 2017;40(1):63-73. https://doi.org/10. $1080 / 07317115.2016 .1201714$.

Cognitive impairment, informed consent, and geriatric issues in the conduction of mental health clinical trials in older adults.

37. Schensul JJ, Robison J, Reyes C, Radda K, Gaztambide $\mathrm{S}$, Disch W. Building interdisciplinary/intersectoral research partnerships for community-based mental health research with older minority adults. Am J Community Psychol. 2006;38(1-2):23-5. https://doi. org/10.1007/s10464-006-9059-y.

38. Schlernitzauer M, Bierhals AJ, Geary MD, Prigerson HG, Stack JA, Miller MD, et al. Recruitment methods for intervention research in bereavement-related depression: five years' experience. Am J Geriatr Psychiatry. 1998;6(1):67-74. https://doi.org/10.1097/00019442199802000-00009.

39. Thompson MG, Heller K, Rody CA. Recruitment challenges in studying late-life depression: do community samples adequately represent depressed older adults? Psychol Aging. 1994;9(1):121-5. https://doi.org/10. 1037/0882-7974.9.1.121.

40. Walaszek A. Clinical ethics issues in geriatric psychiatry. Psychiatr Clin North Am. 2009;32(2):343-59. https:// doi.org/10.1016/j.psc.2009.02.004.

41. Wittink MN, Oslin D, Knott KA, Coyne JC, Gallo JJ, Zubritsky C. Personal characteristics and depressionrelated attitudes of older adults and participation in stages of implementation of a multi-site effectiveness trial (PRISM-E). Int J Geriatr Psychiatry.

2005;20(10):927-37. https://doi.org/10.1002/gps 1386.

42. Woodall A, Morgan C, Sloan C, Howard L. Barriers to participation in mental health research: are there specific gender, ethnicity and age related barriers? BMC Psychiatry. 2010;10:103. https://doi.org/10.1186/ 1471-244X-10-103.

43. Adams M, Caffrey L, McKevitt C. Barriers and opportunities for enhancing patient recruitment and retention in clinical research: findings from an interview study in an NHS academic health science centre. Health Res Policy Syst. 2015;13. https://doi.org/10. 1186/1478-4505-13-8.

44. Borschmann R, Patterson S, Poovendran D, Wilson D, Weaver $\mathrm{T}$. Influences on recruitment to randomised controlled trials in mental health settings in England: a national cross-sectional survey of researchers working for the Mental Health Research Network. BMC Med Res Methodol. 2014;14:23. https://doi.org/10.1186/ 1471-2288-14-23.

45. Bucci S, Butcher I, Hartley S, Neil ST, Mulligan J, Haddock G. Barriers and facilitators to recruitment in mental health services: care coordinators' expectations and experience of referring to a psychosis research trial. Psychol Psychother Theory Res Pract. 2015;88(3):33550. https://doi.org/10.1111/papt.12042.

46. Patel MX, Doku V, Tennakoon L. Challenges in recruitment of research participants. Adv Psychiatr Treat. 2003;9(3):229-38. https://doi.org/10.1192/apt.9.3. 229.

47. Patterson S, Kramo K, Soteriou T, Crawford MJ. The great divide: a qualitative investigation of factors influencing researcher access to potential randomised controlled trial participants in mental health settings. J Ment Health. 2010;19(6):532-41. https://doi.org/10. 3109/09638237.2010.520367.

48. Mason VL, Shaw A, Wiles NJ, Mulligan J, Peters T, Sharp D, et al. GPs' experiences of primary care mental health research: a qualitative study of the barriers to recruitment. Fam Pract. 2007;24(5):518-25. https:// doi.org/10.1093/fampra/cmm047.

49. Ross S, Grant A, Counsell C, Gillespie W, Russell I, Prescott R. Barriers to participation in randomised controlled trials: a systematic review. J Clin Epidemiol. 1999;52(12):1143-56. https://doi.org/10.1016/ S0895-4356(99)00141-9.

50. Howard L, de Salis I, Tomlin Z, Thornicroft G, Donovan J. Why is recruitment to trials difficult? An investigation into recruitment difficulties in an RCT of supported employment in patients with severe mental illness. Contemp Clin Trials. 2009;30(1):40-6. https:// doi.org/10.1016/j.cct.2008.07.007.

51. Jones H, Cipriani A. Barriers and incentives to recruitment in mental health clinical trials. Evid Based Ment Health. 2019;22(2):49-50. https://doi.org/10.1136/ ebmental-2019-300090.

52. McDermott MM, Newman AB. Preserving clinical trial integrity during the coronavirus pandemic. JAMA. 2020. https://doi.org/10.1001/jama.2020.4689.

53. Friedman DB, Foster C, Bergeron CD, Tanner A, Kim S$\mathrm{H}$. A qualitative study of recruitment barriers, motivators, and community-based strategies for increasing clinical trials participation among rural and urban populations. Am J Health Promot. 2015;29(5):332-8. https://doi.org/10.4278/ajhp.130514-QUAL-247.

54. Waheed W, Hughes-Morley A, Woodham A, Allen G, Bower P. Overcoming barriers to recruiting ethnic minorities to mental health research: a typology of recruitment strategies. BMC Psychiatry. 2015;15:101. https://doi.org/10.1186/s12888-015-0484-z.

55. Chang TE, Brill CD, Traeger L, Andres Bedoya C, Inamori A, Hagan PN, et al. Association of race, ethnicity and language with participation in mental health research among adult patients in primary care. J Immigr Minor Health. 2015;17(6):1660-9. https:// doi.org/10.1007/s10903-014-0130-8.

56. George S, Duran N, Norris K. A systematic review of barriers and facilitators to minority research participation among African Americans, Latinos, Asian Americans, and Pacific Islanders. Am J Public Health. 2014;104(2):e16-31. https://doi.org/10.2105/AJPH. 2013.301706. 
57. Mohr DC, Ho J, Duffecy J, Baron KG, Lehman KA, Jin L, et al. Perceived barriers to psychological treatments and their relationship to depression. J Clin Psychol. 2010;66(4):394-409. https://doi.org/10.1002/jclp. 20659.

58. Wainberg ML, Scorza P, Shultz JM, Helpman L, Mootz JJ, Johnson KA, et al. Challenges and opportunities in global mental health: a research-to-practice perspective. Curr Psychiatry Rep. 2017;19(5):28. https://doi. org/10.1007/s11920-017-0780-Z.

59. Heintzman J, Gold R, Krist A, Crosson J, Likumahuwa $\mathrm{S}$, DeVoe JE. Practice-based research networks (PBRNs) are promising laboratories for conducting dissemination and implementation research. J Am Board Fam Med JABFM. 2014;27(6):759-62. https://doi.org/10. 3122/jabfm.2014.06.140092.

60. Carey TS, Halladay JR, Donahue KE, Cykert S. Practicebased research networks (PBRNs) in the era of integrated delivery systems. J Am Board Fam Med JABFM. 2015;28(5):658-62. https://doi.org/10.3122/jabfm. 2015.05.140353.

61. Posternak MA, Zimmerman M. Therapeutic effect of follow-up assessments on antidepressant and placebo response rates in antidepressant efficacy trials: metaanalysis. Br J Psychiatry. 2007;190(4):287-92. https:// doi.org/10.1192/bjp.bp.106.028555.

62. Mulsant BH, Blumberger DM, Ismail Z, Rabheru $\mathrm{K}$, Rapoport MJ. A systematic approach to the pharmacotherapy of geriatric major depression. Clin Geriatr Med. 2014;30(3):517-34. https://doi.org/10.1016/j. cger.2014.05.002.

63. Alexopoulos GS, Reynolds CF, Bruce ML, et al. Reducing suicidal ideation and depression in older primary care patients: 24-month outcomes of the prospect study. Am J Psychiatry. 2009;166(8):882-90. https:// doi.org/10.1176/appi.ajp.2009.08121779.

64. Dunn LB, Lindamer LA, Palmer BW, Schneiderman LJ, Jeste DV. Enhancing comprehension of consent for research in older patients with psychosis: a randomized study of a novel consent procedure. Am J Psychiatry. 2001;158(11):1911-3. https://doi.org/10.1176/appi. ajp.158.11.1911.

65. Hughes TB, Varma VR, Pettigrew C, Albert MS. African Americans and clinical research: evidence concerning barriers and facilitators to participation and recruitment recommendations. The Gerontologist. 2017;57(2):348-58. https://doi.org/10.1093/geront/ gnv118.

66. Zullino D, Conus P, Borgeat F, Bonsack C. Readiness to participate in psychiatric research. Can J Psychiatr.
2003;48(7):480-4. https://doi.org/10.1177/ 070674370304800709.

67. Simning A, van Wijngaarden E, Conwell Y. Overcoming recruitment barriers in urban older adults residing in congregate living facilities. Psychiatry J. 2015;2015:1-8. https://doi.org/10.1155/2015/ 824672 .

68. Tamariz L, Palacio A, Robert M, Marcus EN. Improving the informed consent process for research subjects with low literacy: a systematic review. J Gen Intern Med. 2013;28(1):121-6. https://doi.org/10.1007/s11606012-2133-2.

69. Nishimura A, Carey J, Erwin PJ, Tilburt JC, Murad MH, McCormick JB. Improving understanding in the research informed consent process: a systematic review of 54 interventions tested in randomized control trials. BMC Med Ethics. 2013;14:28. https://doi.org/10. 1186/1472-6939-14-28.

70. Sherrill JT, Frank E, Geary M, Stack JA, Reynolds CF. Psychoeducational workshops for elderly patients with recurrent major depression and their families. Psychiatr Serv. 1997;48(1):76-81. https://doi.org/10.1176/ps. 48.1.76.

71. Hart AR, Dillard R, Perkins MM, Vaughan CP, Kinlaw $\mathrm{K}$, McKay JL, et al. The DREAMS team: creating community partnerships through research advocacy training for diverse older adults. Educ Gerontol. 2017;43(9):440-50. https://doi.org/10.1080/ 03601277.2017 .1321449$.

72. Dowrick C, Bower P, Chew-Graham C, Lovell K, Edwards S, Lamb J, et al. Evaluating a complex model designed to increase access to high quality primary mental health care for under-served groups: a multimethod study. BMC Health Serv Res. 2016;16:58. https://doi.org/10.1186/s12913-016-1298-5.

73. Kogan JN, Bauer MS, Dennehy EB, Miklowitz DJ, Gonzalez JM, Thompson PM, et al. Increasing minority research participation through collaboration with community outpatient clinics: the STEP-BD Community Partners Experience. Clin Trials. 2009;6(4):34454. https://doi.org/10.1177/1740774509338427.

\section{Publisher's Note}

Springer Nature remains neutral with regard to jurisdictional claims in published maps and institutional affiliations. 